\title{
Simplified detection of the asymmetric polymerase chain reaction-amplified DNA and its application in the target identification
}

\author{
G Suhasa, Savithri Bhat* \\ Department of Biotechnology, BMS College of Engineering, Bengaluru, Karnataka, India
}

\begin{tabular}{l}
\hline ARTICLE INFO \\
\hline Article history: \\
Received on: October 13, 2017 \\
Accepted on: November 28, 2017 \\
Available online: February 17, 2018
\end{tabular}

Key words:

Polymerase chain reaction,

Agarose gel electrophoresis,

Asymmetric,

Loop-mediated isothermal

amplification,

Fluorometry

\begin{abstract}
The nucleic acid amplification methods such as the polymerase chain reaction (PCR) and the loop-mediated isothermal amplification rely on the detection of the amplified products by means of agarose gel electrophoresis. The detection of the amplified target DNA in an asymmetric PCR was simplified by carrying out the probe hybridization, polymerization, and the subsequent measurement of fluorescence of the double-stranded target DNA (dsDNA) using the Qubit ${ }^{\circledR}$ dsDNA BR Assay Kit. This method was validated by detecting the lacZ gene that is present in the pUC 18 plasmid as well as by detecting the CDH13 gene that is present in the human genomic DNA.
\end{abstract}

\section{INTRODUCTION}

The amplified product of the polymerase chain reaction (PCR) is generally detected by carrying out the agarose gel electrophoresis. It consists of the separation of the DNA fragments by applying an electric field to the agarose gel which acts as a sieving medium [1]. However, the preparation of the agarose gel and the subsequent electrophoresis process is cumbersome and takes 1-2 h. The dye toluidine blue can be used in the detection and the estimation of the PCR-amplified DNA, but the interference of the dNTPs and the primers cannot be neglected [2]. The use of the methyl green for the estimation of the DNA is time consuming $[3,4]$. The detection of the amplified nucleic acid based on the change in the $\mathrm{pH}$ during the course of the reaction requires complex and expensive instrumentation [5]. Although the $\mathrm{pH}$-sensitive dyes can be used in the detection of the change in the $\mathrm{pH}$, the target DNA should be greater than $500 \mathrm{bp}$ [6]. The color change produced by the reaction of ferroin reagent (1, 10-phenanthroline) and the pyrophosphate that is released as a byproduct during the nucleic acid amplification is hardly visible to the naked eyes [7]. The GeneFinder ${ }^{\mathrm{TM}}$ nucleic acid dye can be used to detect the presence of amplified DNA in the PCR or loop-mediated isothermal amplification [8] but is expensive.

*Corresponding Author:

Savithri Bhat,

Department of Biotechnology, BMS College of Engineering,

Bengaluru - 560 019, Karnataka, India.

Email: hod.bt@bmsce.ac.in
The above said colorimetric methods are not specific to the amplified target DNA. It detects the production of any amplified DNA resulting due to the mispriming or the suboptimal reaction conditions.

The PCR produces double-stranded target amplicon [9]. The asymmetric PCR (aPCR) that relies on the differing primer concentration [10] or the dissimilar Tm of the primers [11] has been used to produce the targetspecific single-stranded DNA (ssDNA) along with the double-stranded target DNA (dsDNA). The products of the aPCR can be detected by the methods such as the probe hybridization and fluorescence polarization [12], solid phase assay [13], or gold nanoparticle-based colorimetric assay $[14,15]$.

The hybridization of the target-specific probe to the single-stranded aPCR product and the subsequent extension/polymerization of the probe produces dsDNA which can be detected as an increase in fluorescence intensity using Qubit ${ }^{\circledR}$ dsDNA BR Assay Kit. The conversion of the ssDNA to the dsDNA and an increase in the amount of the dsDNA can also be seen as the mobility shift of the ssDNA by the gel electrophoresis. The utility of this method has been verified in the present work by confirming the presence of lacZ gene in the pUC18 plasmid as well as by detecting the presence of CDH13 gene in the human genome.

\section{MATERIALS AND METHODS}

The pUC18 plasmid and the human genomic DNA were purchased from Merck Genei (Bangalore, India). The forward and reverse PCR primers and the probe were purchased from Sigma-Aldrich 
(Bangalore, India). The 100 bp DNA molecular weight marker was from BR Biochem Life Sciences Pvt. Ltd. (New Delhi, India). PCR/ aPCR was carried out in the "Applied Biosystems 2720" thermal cycler. The PCR reagents were from New England Biolabs (Gurgaon, India). The Qubit ${ }^{\mathbb{R}}$ dsDNA BR Assay Kit was purchased from Thermo Fisher Scientific (Bangalore, India).

\subsection{PCR Based on Equimolar Primers}

The target-specific dsDNA amplicon was synthesized by the PCR that had equimolar concentration of the forward and the reverse primers ( $0.1 \mathrm{uM}$ each primer). A total of 35 cycles of the template denaturation, primer annealing, and primer extension were carried out. The thermal cycling conditions were identical to that of the corresponding aPCR as described below. This PCR was used as a positive control in the present work.

\subsection{Detection of the lacZ Gene Present in the pUC18 Plasmid by the Conventional aPCR by Varying the Primer Concentration and by the Late Priming aPCR (LP-aPCR)}

The target DNA (partial lacZ gene) to be amplified was $500 \mathrm{bp}$. The conventional aPCR contained $20 \mathrm{pmol}$ of the excess primer and $1 \mathrm{pmol}$ of the limiting primer. The base sequence of the forward primer and the reverse primer was, respectively, 5'-CTATGCGGCATCAGAGCAG-3' and 5'-GATTCATTAATGCAGCTGGCACG-3'. The reaction volume was $50 \mathrm{ul}$ and contained $200 \mathrm{uM}$ dNTPs, $8 \mathrm{ng}$ pUC18, and 5 units of Taq DNA polymerase. The initial denaturation was at $94^{\circ} \mathrm{C}$ for $2 \mathrm{~min}$, and 35 cycles of denaturation at $94^{\circ} \mathrm{C}$ for $30 \mathrm{~s}$, annealing at $52^{\circ} \mathrm{C}$ for $30 \mathrm{~s}$, and primer extension at $68^{\circ} \mathrm{C}$ for $30 \mathrm{~s}$ were carried out, and the final extension was at $68^{\circ} \mathrm{C}$ for $5 \mathrm{~min}$. $15 \mathrm{ul}$ of the reaction volume was then added to each of the three tubes that were labeled as "without probe," "with complementary probe," and "with non-complementary probe." 10 pmol of the specific probe was added to the reaction, and one cycle of denaturation at $94^{\circ} \mathrm{C}$ for $30 \mathrm{~s}$, annealing at $53^{\circ} \mathrm{C}$ for $30 \mathrm{~s}$, and probe extension at $68^{\circ} \mathrm{C}$ for $15 \mathrm{~min}$ was carried out. The base sequence of the complementary and the non-complementary probes was, respectively, 5'-AGGGGGATGT GCTGCAAGGCGATTAAGTTGGGTAACGCCAGGGTTTTCC CAGTCACGACGTTGTAA-3' and 5'-GGATCACCTCCTTACCTT AAAGAAGC-3'. 5 ul of the postreaction mix was loaded on $3 \%$ agarose gel, electrophoresed, and visualized under the UV light. The detection of the amplified target DNA based on the fluorescence was carried out using the Qubit ${ }^{\mathbb{B}}$ fluorometer and the Qubit ${ }^{\mathbb{B}}$ dsDNA BR Assay Kit according to the manufacturer's instructions.

The LP-aPCR was carried out using 5 pmol each of the forward and the reverse primer and 27 cycles of denaturation at $94^{\circ} \mathrm{C}$ for $30 \mathrm{~s}$, annealing at $55^{\circ} \mathrm{C}$ for $30 \mathrm{~s}$, and primer extension at $68^{\circ} \mathrm{C}$ for $30 \mathrm{~s}$. In the second step, $30 \mathrm{pmol}(3 \mathrm{ul})$ of the excess primer was added to the reaction, and further, eight cycles were carried out. Finally, the probe was added and extended as said above. The postreaction mix was electrophoresed, and the Qubit fluorescence-based detection of the amplified target DNA was carried out according to the manufacturer's instructions.

\subsection{Detection of the CDH13 Gene Present in the Human Genomic DNA by the Conventional aPCR by Varying the Primer Concentration And by the LP-aPCR}

The target DNA (partial CDH13 gene) to be amplified was 416 bp of the CDH13 gene present in the human genomic DNA. The conventional aPCR contained $30 \mathrm{pmol}$ of the excess primer and $5 \mathrm{pmol}$ of the limiting primer. The base sequence of the forward primer and the reverse primer was, respectively, 5'-GGATCTCAGGGTGGGTGGCAATGCT-3' and 5'-GAAAGGCAAGCTACCAGAAGCCCCAA-3'. The reaction volume was $50 \mathrm{ul}$ and contained $200 \mathrm{uM}$ dNTPs, $300 \mathrm{ng}$ of human genomic DNA, and 5 units of Taq DNA polymerase. The initial denaturation was at $94^{\circ} \mathrm{C}$ for $2 \mathrm{~min}$, and 35 cycles of denaturation at $94^{\circ} \mathrm{C}$ for $30 \mathrm{~s}$, annealing at $63^{\circ} \mathrm{C}$ for $30 \mathrm{~s}$, and primer extension at $68^{\circ} \mathrm{C}$ for $30 \mathrm{~s}$ were carried out, and the final extension was at $68^{\circ} \mathrm{C}$ for $5 \mathrm{~min} .15 \mathrm{ul}$ of the reaction volume was added to each of the three tubes that were labeled as "without probe," "with complementary probe," and "with non-complementary probe." 10 pmol of the specific probe was then added and extended using one cycle of denaturation at $94^{\circ} \mathrm{C}$ for $30 \mathrm{~s}$, annealing at $60^{\circ} \mathrm{C}$ for $30 \mathrm{~s}$, and probe extension at $68^{\circ} \mathrm{C}$ for $15 \mathrm{~min}$. The base sequence of the complementary and the non-complementary probes was, respectively, 5'-AATAAATCTCCC CGTTCTCAACT CCTGTGTG-3' and 5'-AGGGGGA TGTGCTG CAAGGCGATTAA GTTGGGTAACGCCAGGGTTTTCCC AGTCACGACGTTGTAA-3'. The postreaction mix was electrophoresed, and the Qubit fluorescence-based detection of the amplified target DNA was carried out according to the manufacturer's instructions.

The LP-aPCR was carried out using 5 pmol each of the forward and the reverse primer and 27 cycles of denaturation at $94^{\circ} \mathrm{C}$ for $30 \mathrm{~s}$, annealing at $63^{\circ} \mathrm{C}$ for $30 \mathrm{~s}$, and primer extension at $68^{\circ} \mathrm{C}$ for $30 \mathrm{~s}$. In the second step, $30 \mathrm{pmol}(3 \mathrm{ul})$ of the excess primer was added to the reaction, and further, eight cycles were carried out. Finally, the probe was added and extended as said above. The postreaction mix was electrophoresed, and the Qubit fluorescence-based detection of the amplified target DNA was carried out according to the manufacturer's instructions.

\section{RESULTS AND DISCUSSION}

The conventional aPCR based on the excess and the limiting concentration of the forward and the reverse primers produce the targetspecific ssDNA by the repeated extension of the respective primer that is present in excess. The LP-aPCR produces an exponentially amplified double-stranded DNA in 27 cycles of denaturation, annealing, and extension. The addition of an appropriate excess primer in the next step linearly amplifies the preferred strand in further eight cycles. Using the pUC18 plasmid as the template, the conventional aPCR based on the excess and the limiting primer concentration produced multiple non-target dsDNA [Figure 1a, L2] and liberated no ssDNA [Figure 1a, L3] under the conditions similar to that of the LP-aPCR. A definite ssDNA was synthesized when the appropriate forward or the reverse primer was added in excess in the second step of the LP-aPCR [Figure 1b, L2, and L3].

The addition of the target-specific probe hybridizes to the ssDNA that was synthesized by the second step of the LP-aPCR and gets polymerized with the help of the DNA polymerase that is left over in the reaction or by adding the fresh enzyme. The amount of the dsDNA is thus increased by the probe extension post-aPCR. The synthesis of the dsDNA by the probe extension is seen as the mobility shift of the ssDNA on the agarose gel [L3 of the Figures 2a and 3a].

The increase in the amount of the dsDNA due to the probe extension is easily detected with Qubit fluorometer using the Qubit ${ }^{\circledR}$ dsDNA BR Assay Kit [Figures $2 b$ and $3 b$ ]. The use of the unrelated noncomplementary probe does not affect the total amount of the dsDNA in the reaction and is identical to the no probe control as seen by the gel electrophoresis [L2 and L4 of the Figures 2a and 3a] as well as 


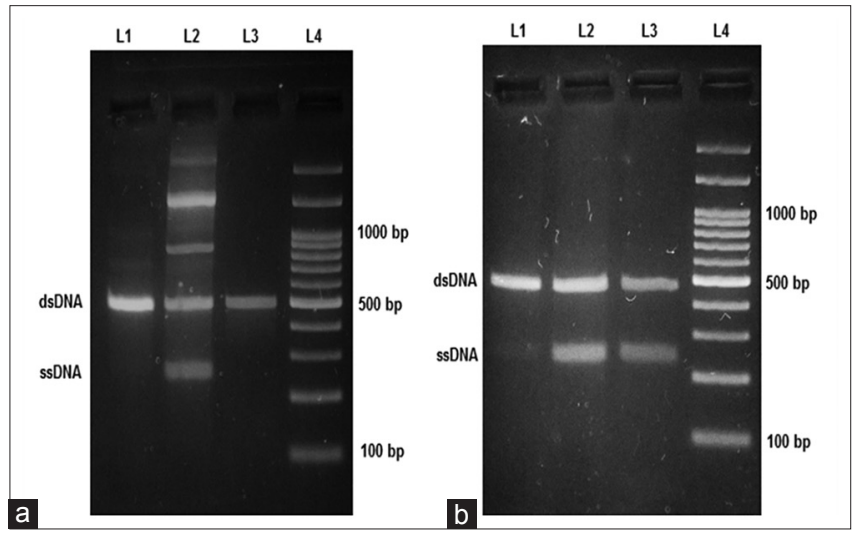

Figure 1: Synthesis of the single-stranded DNA by the conventional asymmetric polymerase chain reaction (a) and by the late priming asymmetric polymerase chain reaction (b). L1 - The target-specific dsDNA produced by the standard polymerase chain reaction (PCR) using equimolar concentration of forward and reverse primers. L2 - The target-specific dsDNA and ssDNA produced by the conventional asymmetric PCR (aPCR) (a) or the late priming aPCR (LP-aPCR) (b) using an excess of the respective forward primer.

L3 - the target-specific dsDNA and ssDNA produced by the conventional aPCR (a) or the LP-aPCR (b) using an excess of the respective reverse primer.

\section{L4 - 100 bp DNA ladder.}

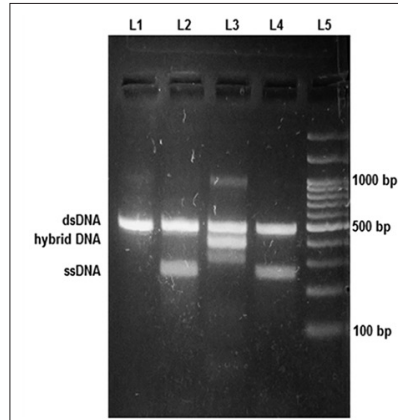

a

Figure 2: The extension of the probe that is hybridized to the late priming asymmetric polymerase chain reaction (LP-aPCR) product and the detection of the amplified lacZ gene fragment by the agarose gel electrophoresis (a) or by the Qubit-based assay (b). A. L1 - sPCR. L2 - NPA. L3 - MP. L4 - MMP. L5 - 100 bp DNA ladder. B. The Qubit assay was used to measure the dsDNA concentration after carrying out the probe extension. sPCR - the standard PCR using equimolar concentration of forward and reverse primers. NPA - the LP-aPCR products were not hybridized with any probe. MMP - the LP-aPCR products were treated with an unrelated mismatched probe. MP - the LP-aPCR products were hybridized with a target-specific complementary probe and were extended.

in Qubit-based assay [Figures $2 \mathrm{~b}$ and 3b]. Hence, the presence of the amplified target DNA is detected in more simplified way using the Qubit-based fluorescence assay without carrying out the agarose gel electrophoresis.

The target-specific probe hybridizes to the single-stranded LP-aPCR product, and the extension of the probe forms $321 \mathrm{bp}$ of the targeted lacZ gene fragment leaving 179 bases of the single-stranded LP-aPCR product unaltered. This hybrid DNA has a higher mobility in 3\% agarose gel than the target-specific dsDNA of size 500 bp that was formed in

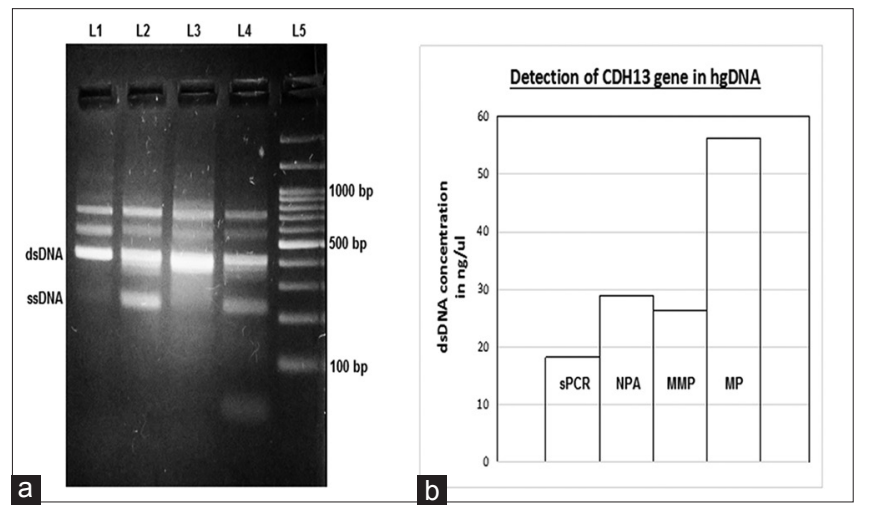

Figure 3: The extension of the probe that is hybridized to the late priming asymmetric polymerase chain reaction (LP-aPCR) product and the detection of the amplified $\mathrm{CDH} 13$ gene fragment by the agarose gel electrophoresis (a) or by the Qubit-based assay (b). A. L1 - sPCR. L2 - NPA. L3 - MP. L4 - MMP. L5 - 100 bp DNA ladder. (b) the Qubit assay was used to measure the dsDNA concentration after carrying out the probe extension. SPCR - the standard PCR using equimolar concentration of forward and reverse primers. NPA - the LP-aPCR products were not hybridized with any probe. MMP - the LP-aPCR products were treated with an unrelated mismatched probe. MP - the LP-aPCR products were hybridized with a target-specific complementary probe and were extended.

the initial step of LP-aPCR [Figure 2a, L3]. It is seen that there is an extra DNA fragment that has a still higher mobility in the agarose gel [Figure 2a, L3]. This was found to be a dsDNA that was a by-product formed (product size $=321 \mathrm{bp}$ ) during the probe extension step by the extension of the probe and the reverse primer (result not shown).

The gene CDH13 contains an internal Alu transposon [16]. The size of the Alu DNA is $308 \mathrm{bp}$ and the size of the CDH13 gene fragment lacking the Alu insertion is $416 \mathrm{bp}$. The human genome contains a diverse expression of the Alu transposon. The genotype of the CDH13 gene is affected by the presence or the absence of the Alu transposon. Hence, the DNA fragment that is amplified by the primers that target the $\mathrm{CDH} 13$ gene results in the synthesis of DNA fragments of multiple sizes. The $416 \mathrm{bp}$ DNA fragment gets amplified from the human genomic DNA containing the CDH13 gene without the Alu transposon. The amplified DNA fragment of size $\sim 700 \mathrm{bp}$ is due to the human $\mathrm{CDH} 13$ gene containing the Alu transposon. The amplified DNA fragment of size $\sim 600$ bp DNA fragment is the hybrid DNA containing certain parts of the amplified $416 \mathrm{bp}$ and the $\sim 700 \mathrm{bp}$ DNA fragments [Figure 3a]. After the completion of the LP-aPCR, the targetspecific probe hybridizes to the single-stranded LP-aPCR product, and the extension of the probe forms $371 \mathrm{bp}$ of the targeted CDH13 gene fragment, leaving 45 bases of the single-stranded LP-aPCR product unaltered. This hybrid DNA has a mobility in 3\% agarose gel similar to that of the target-specific dsDNA of size $416 \mathrm{bp}$ that was formed in the initial step of LP-aPCR [Figure 3, L3].

The conventional aPCR is based on the differing concentration of the forward and the reverse primers and requires the optimized ratio of the amount of the excess and the limiting primer. This limitation is overcome using the LATE-PCR [17]. It corrects the primer concentration-dependent $\mathrm{Tm}$ by incorporating the nucleotides to the 5 ' end of the limiting primer. This results in the excess and the limiting primers of identical melting temperature and thereby eliminates the differential annealing characteristics of the primers. We have carried 
out the synthesis of ssDNA using a two-step aPCR and have termed it as the LP-aPCR. In the first step, an equimolar concentration of the forward and the reverse primer produces the dsDNA in 27 cycles of the template denaturation, primer annealing, and primer extension. Then, an excess of the respective forward or the reverse primer is added to the reaction to synthesize the corresponding ssDNA in further eight cycles. Since there is no addition of the limiting primer in the LP-aPCR, its primer concentration-dependent Tm remains unimportant.

The detection of the PCR product by the electrophoresis in an ethidium bromide stained gel is less sensitive than the spectrofluorometric estimation of the DNA in a solution [18]. The use of the dsDNA binding dyes such as that of the dsDNA BR Assay Kit can quantitate the PCR product with an increased sensitivity.

The spectrofluorometric quantitation of the PCR product reveals the presence or the absence of the dsDNA in the post-PCR mix. It cannot conclusively show whether the synthesized dsDNA is the expected target since the PCR can also produce the dsDNA due to mispriming or due to the deviation of the PCR from the optimized conditions. By combining the detection of the LP-aPCR product that is extended by a specific probe with the Qubit assay, the presence of the specific target can be determined using the fluorometer.

\section{CONCLUSION}

The above said method of the LP-aPCR followed by the probe extension, and the subsequent Qubit assay helps in the rapid detection of the amplified DNA target without carrying out the agarose gel electrophoresis. This method has a potential use in the detection of DNA polymorphism. The commercially available kit such as the "Type-it ${ }^{\circledR}$ mutation detect PCR Kit" marketed by Qiagen rely on the use of allele-specific PCR that helps in a sensitive mutation detection whereas the "Surveyor ${ }^{\circledR}$ mutation detection PCR kit" marketed by Integrated DNA Technologies (IDT) rely on the hybridization of the two PCR products (obtained from the sample with or without the mutation) and the subsequent cleavage at the mismatched base using the surveyor nuclease. Both of these methods detect the amplified PCR product by the size-based separation such as the conventional agarose gel electrophoresis or by the capillary gel electrophoresis. Hence, the newly developed method as described in this paper might be used as a rapid and less time-consuming non-electrophoretic detection of DNA mutation.

The detection of the target-specific DNA using the Qubit fluorometer and the Qubit ${ }^{\circledR}$ dsDNA BR Assay Kit is rapid and cost-effective $(<\$ 1.5 /$ - per sample containing the dye). As per our knowledge, this is the first report describing the use of the Qubit-based assay in the simplified detection of the target-specific DNA that is amplified by an aPCR.

\section{ACKNOWLEDGMENT}

The authors thank TEQIP (Grant No.16-5/2013-TS.VII (Pt.)), BMSCE, and the VGST, KFIST-1 (GRD 361) for providing the fund to carry out the current research. We also thank Dr. Arindam Dhali, National Institute of Animal Nutrition and Physiology (NIANP) and the Director, National Institute of Animal Nutrition and Physiology (NIANP), Bangalore, for permitting to use the Qubit fluorometer with the necessary reagents and consumables.

\section{REFERENCES}

1. Green MR, Sambrook J. Molecular Cloning a Laboratory Manual. $4^{\text {th }}$ ed. USA: Cold Spring Harbour; 2012.

2. Killeen AA. A visible spectrophotometric assay for submicrogram quantities of DNA, including PCR-amplified DNA. Microchem J 1995;52:333-40.

3. Peters DL, Dahmus ME. A method of DNA quantitation for localization of DNA in metrizamide gradients. Anal Biochem 1979;93:306-11.

4. Sinicropi D, Baker DL, Prince WS, Shiffer K, Shak S. Colorimetric determination of DNase I activity with a DNA-methyl green substrate. Anal Biochem 1994;222:351-8.

5. Toumazou C, Shepherd LM, Reed SC, Chen GI, Patel A, Garner DM, et al. Simultaneous DNA amplification and detection using a $\mathrm{pH}-$ sensing semiconductor system. Nat Methods 2013;10:641-6.

6. Tanner NA, Zhang Y, Evans TC Jr. Visual detection of isothermal nucleic acid amplification using $\mathrm{pH}$-sensitive dyes. Biotechniques 2015;58:59-68.

7. Lee K, Kim K. A colorimetric confirmation method for DNA amplification in PCR and its application to the detection of Giardia lamblia cysts. Biotechnol Lett 2003;25:1739-42.

8. Almasi MA, Erfan Manesh M, Jafary H, Dehabadi SM. Visual detection of potato leafroll virus by loop-mediated isothermal amplification of DNA with the geneFinder ${ }^{\mathrm{TM}}$ dye. J Virol Methods 2013;192:51-4.

9. Mullis K, Faloona F, Scharf S, Saiki R, Horn G, Erlich H, et al. Specific enzymatic amplification of DNA in vitro: The polymerase chain reaction. Cold Spring Harb Symp Quant Biol 1986;51 Pt 1:263-73.

10. Gyllensten UB, Erlich HA. Generation of single-stranded DNA by the polymerase chain reaction and its application to direct sequencing of the HLA-DQA locus. Proc Natl Acad Sci U S A 1988;85:7652-6.

11. Mazars GR, Moyret C, Jeanteur P, Theillet CG. Direct sequencing by thermal asymmetric PCR. Nucleic Acids Res 1991;19:4783.

12. Fujii T, Ohta M, Kono M, Hoshina S, Fukuhara K, Tsuruoka M, et al. Rapid detection of the gene of Legionella pneumophila using the fluorescence polarization with the asymmetric PCR. Nucleic Acids Symp Ser 1999;42:59-60.

13. Nolasco G, Sequeira Z, Soares C, Mansinho A, Bailey AM, Niblett CL. Asymmetric PCR ELISA: Increased sensitivity and reduced costs for the detection of plant viruses. Eur J Plant Pathol 2002;108:293-8.

14. Deng H, Xu Y, Liu Y, Che Z, Guo H, Shan S, et al. Gold nanoparticles with asymmetric polymerase chain reaction for colorimetric detection of DNA sequence. Anal Chem 2012;84:1253-8.

15. Wang L, Liu Z, Xia X, Huang J. Visual detection of Maize chlorotic mottle virus by asymmetric polymerase chain reaction with unmodified gold nanoparticles as the colorimetric probe. Anal Methods 2016;8:6959-64.

16. Comas D, Plaza S, Calafell F, Sajantila A, Bertranpetit J. Recent insertion of an alu element within a polymorphic human-specific alu insertion. Mol Biol Evol 2001;18:85-8.

17. Sanchez JA, Pierce KE, Rice JE, Wangh LJ. Linear-after-theexponential (LATE)-PCR: An advanced method of asymmetric PCR and its uses in quantitative real-time analysis. Proc Natl Acad Sci U S A 2004;101:1933-8.

18. Bonasera V, Alberti S, Sacchetti A. Protocol for high-sensitivity/long linear-range spectrofluorimetric DNA quantification using ethidium bromide. Biotechniques 2007;43:173-4, 176.

\footnotetext{
How to cite this article:

Suhasa G, Bhat S. Simplified detection of the asymmetric polymerase chain reaction-amplified DNA and its application in the target identification. J App Biol Biotech. 2018;6(2):50-53. DOI: 10.7324/JABB.2018.60208
} 